\title{
Transformations of Border Strips and Schur Function Determinants
}

\author{
WILLIAM Y.C. CHEN \\ GUO-GUANG YAN \\ ARTHUR L.B. YANG \\ Center for Combinatorics, LPMC, Nankai University, Tianjin 300071, P. R. China
}

chen@nankai.edu.cn guogyan@eyou.com yang@nankai.edu.cn

Received February 25, 2004; Revised June 21, 2004; Accepted September 8, 2004

\begin{abstract}
We introduce the notion of the cutting strip of an outside decomposition of a skew shape, and show that cutting strips are in one-to-one correspondence with outside decompositions for a given skew shape. Outside decompositions are introduced by Hamel and Goulden and are used to give an identity for the skew Schur function that unifies the determinantal expressions for the skew Schur functions including the Jacobi-Trudi determinant, its dual, the Giambelli determinant and the rim ribbon determinant due to Lascoux and Pragacz. Using cutting strips, one obtains a formula for the number of outside decompositions of a given skew shape. Moreover, one can define the basic transformations which we call the twist transformation among cutting strips, and derive a transformation theorem for the determinantal formula of Hamel and Goulden. The special case of the transformation theorem for the Giambelli identity and the rim ribbon identity was obtained by Lascoux and Pragacz. Our transformation theorem also applies to the supersymmetric skew Schur function.
\end{abstract}

Keywords: Young diagram, border strip, outside decomposition, Schur function determinants, Jacobi-Trudi identity, Giambelli identity, Lascoux-Pragacz identity

\section{Introduction}

We assume that the reader is familiar with the background of the determinantal expressions for the skew Schur function $s_{\lambda / \mu}(X)$ in the variable set $X=\left\{x_{1}, x_{2}, \ldots\right\}$, including the Jacobi-Trudi determinant, its dual, the Giambelli determinant and the Lascoux-Pragacz rim ribbon determinant. Here are a few references $[2,3,5,6,9,10,12,14-16]$.

This paper is motivated by the remarkable discovery of Hamel and Goulden [7] that the four determinantal formulas for the skew Schur functions can be unified through the concept of outside decompositions of a skew shape. For any outside decomposition, Hamel and Goulden derive a determinantal formula with strip Schur functions as entries. Their proof is based on a lattice path construction and the Gessel-Viennot methodology [6]. The first result of this paper is the notion of cutting strips of outside decompositions for a given skew shape. We show that each outside decomposition can be recovered from a strip, which we call the cutting strip. It turns out that the number of outside decompositions follows from the enumeration of cutting strips. Moreover, the cutting strips can be used to give an easier construction of the strips involved in the Hamel-Goulden determinant.

The second result of this paper is a transformation theorem for the Hamel-Goulden determinant. Using cutting strips to represent outside decompositions, one can define the 
basic steps to transform any outside decomposition to another which we call the twist transformations. For the twist transformations on outside decompositions, we give a construction of determinantal operations that transform the determinantal formula for one outside decomposition to the determinantal formula corresponding to any other outside decomposition.

We remark that the arguments in this paper can be carried over to the case of supersymmetric skew Schur functions and the corresponding determinantal formulas.

\section{Cutting strips}

We begin this section with a review of relevant terminology and notation. Let $\lambda$ be a partition of $n$ with at most $k$ parts, i.e., $\lambda=\left(\lambda_{1}, \lambda_{2}, \ldots, \lambda_{k}\right)$ where $\lambda_{1} \geq \lambda_{2} \geq \cdots \geq \lambda_{k} \geq 0$ and $\lambda_{1}+\lambda_{2}+\cdots+\lambda_{k}=n$. The (Young or Ferrers) diagram of $\lambda$ may be defined as the set of points $(i, j) \in \mathbb{Z}^{2}$ such that $1 \leq j \leq \lambda_{i}$ and $1 \leq i \leq k$. It can be represented in the plane by an array of square boxes or cells that is top and left justified with $k$ rows and $\lambda_{i}$ boxes in row $i$. A box $(i, j)$ in the diagram is the box in row $i$ from the top and column $j$ from the left. Given two partitions $\lambda$, $\mu$ with at most $k$ parts, and $\lambda_{i} \geq \mu_{i}$, for $i=1,2, \ldots, k$, then $\lambda / \mu$ is a skew partition, and the diagram of skew shape $\lambda / \mu$ or the skew diagram of $\lambda / \mu$ is defined as the set of points $(i, j) \in \mathbb{Z}^{2}$ such that $\mu_{i}<j \leq \lambda_{i}$ and $1 \leq i \leq k$. In this paper, we will not distinguish a skew partition $\lambda / \mu$ with its diagram. Thus, the conjugate of a skew partition $\lambda / \mu$, which we denote by $\lambda^{\prime} / \mu^{\prime}$, is defined to be the transpose of the skew diagram $\lambda / \mu$.

A (border) strip or a ribbon is a skew diagram with an edgewise connected set of boxes that contains no $2 \times 2$ block of boxes, where two boxes are said to be edgewise connected if they share a common edge. One may impose a natural direction to each strip. The starting box is always the one at the lower left corner. Moreover, for each box that is not the last box in a strip, we say that it goes right if the next box is to its right, otherwise, we say that it goes up.

Recall that the content of a box $(i, j)$ in a skew diagram $\lambda / \mu$ is given by $j-i$, and a diagonal with content $c$ of $\lambda / \mu$ is the set of all the boxes in $\lambda / \mu$ having content $c$.

Let $\lambda / \mu$ be a skew diagram, a border strip decomposition of $\lambda / \mu$ is a partitioning of the boxes of $\lambda / \mu$ into pairwise disjoint strips. Figure 1 gives two examples of border strip decompositions of the skew shape 6653/211. We now come to the crucial definition of an outside decomposition of a skew shape as introduced by Hamel and Goulden [7]. A border strip decomposition of $\lambda / \mu$ is said to be an outside decomposition of $\lambda / \mu$ if every strip in the decomposition has a starting box on the left or bottom perimeter of the diagram and an ending box on the right or top perimeter of the diagram, see figure 1.

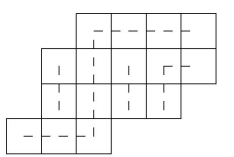

a. A border strip decomposition

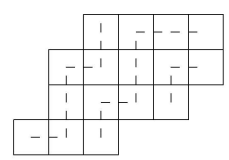

b. An outside decomposition

Figure 1. Border strip decompositions. 
We note that there is slight difference between our description of the outside decomposition and the original definition given by Hamel and Goulden. Since the positions of the strips in an outside decomposition are distinguishable, we may impose a canonical order on the strips. However, in the original treatment of Hamel and Goulden an order of the strips is taken into account and they have noted that any order plays the same role. As we will see later in this paper, we may use the canonical order of the strips by the contents of the ending boxes.

Given an outside decomposition $\Pi$ of a skew diagram $\lambda / \mu$, and a box $(i, j)$ in $\lambda / \mu$, then there is a strip $\theta$ in $\Pi$ that contains $(i, j)$, and we say that the box $(i, j)$ goes right or goes up in $\lambda / \mu$ if it goes right or goes up in $\theta$. When $(i, j)$ is the ending box of $\theta$, then it is on the top or the right perimeter of $\lambda / \mu$, and we say that $(i, j)$ goes up in $\lambda / \mu$ if it is on the top perimeter of $\lambda / \mu$ (including the case when it is a corner of $\lambda / \mu)$, and $(i, j)$ goes right in $\lambda / \mu$ if it is on the right perimeter of $\lambda / \mu$. The directions of the boxes are important owing to the following nested property.

Nested Property (Hamel and Goulden [7]) The strips in any outside decomposition of $\lambda / \mu$ are nested in the sense that the boxes in the same diagonal of $\lambda / \mu$ all go up or all go right.

An immediate consequence of the above nested property is that the contents of the ending (or starting) boxes of the strips in an outside decomposition are different. Hence we may use the contents of the ending boxes to order the strips in an outside decomposition. If there are $m$ strips in $\Pi$, we will denote $\Pi$ by $\Pi=\left(\theta_{1}, \theta_{2}, \ldots, \theta_{m}\right)$, where $\theta_{1}$ is the strip whose ending box has the largest content, etc. In this notation, the outside decomposition of 6653/221 given in figure 1(b) may be denoted by $(4221 / 11,21,3322 / 211)$.

Let $\lambda / \mu$ be a skew diagram. Recall that a block of $\lambda / \mu$ of a skew shape is an edgewise connected component. If a skew shape $\lambda / \mu$ can be decomposed into $k$ blocks $B_{1}, B_{2}, \ldots, B_{k}$, then an outside decomposition $\Pi$ of $\lambda / \mu$ can be decomposed into $\Pi_{1}, \Pi_{2}, \ldots, \Pi_{k}$, where each $\Pi_{i}$ is an outside decomposition of $B_{i}, i=1,2, \ldots, k$. Therefore, without loss of generality one can only deal with outside decompositions of edgewise connected skew shapes. In the same vein, skew Schur function with disconnected shapes can be expressed as a product of skew Schur functions with connected shapes.

Based on the nested property, we are now ready to give construction of the cutting strip of an outside decomposition of an edgewise connected skew shape $\lambda / \mu$.

Definition 2.1 Let $\Pi$ be an outside decomposition of an edgewise connected skew shape $\lambda / \mu$. Suppoese that $\lambda / \mu$ has $d$ diagonals. The cutting strip $T$ of $\Pi$ is given by the rule: for $i=1,2, \ldots, d-1$, the $i$-th box in $T$ goes right or goes up according to whether the boxes in the $i$-th diagonal of $\lambda / \mu$ go right or go up with respect to $\Pi$.

Conversely, for any border strip with $d$ boxes, we may reconstruct the outside decomposition $\Pi$ by peeling off $\lambda / \mu$ while sliding the cutting strip along the diagonals. This operation can be visualized by figure 2 .

Thus, we obtain the following correspondence. 

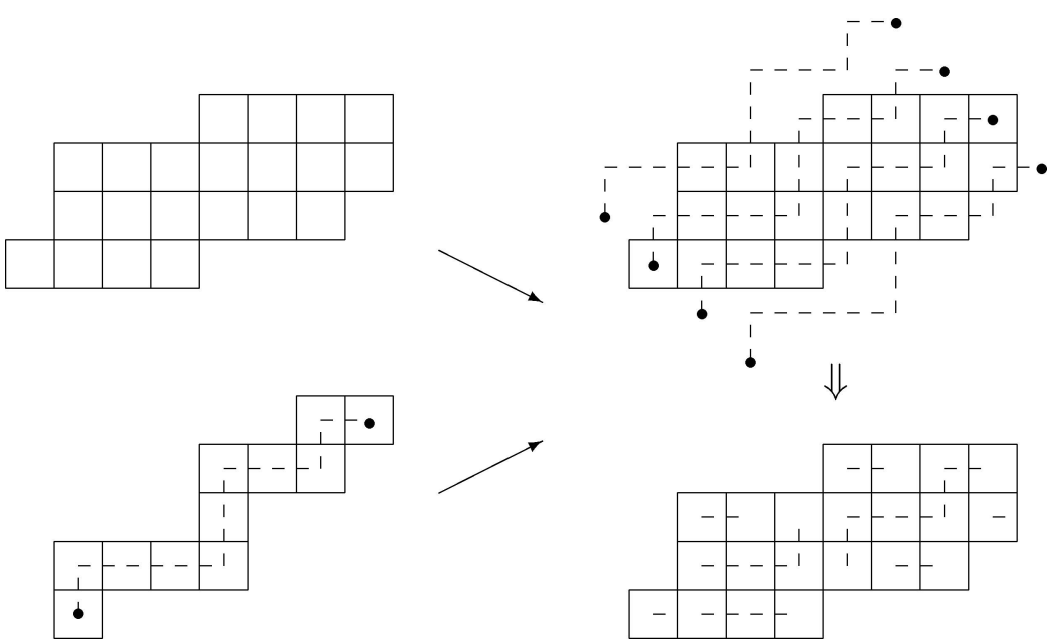

Figure 2. The cutting strip of an outside decomposition.

Theorem 2.2 If $\lambda / \mu$ is an edgewise connected skew shape with d diagonals, then there is a one-to-one correspondence between the outside decompositions of $\lambda / \mu$ and border strips with d boxes.

The above correspondence leads to the following formula for the number of outside decompositions of an edgewise connected skew shape.

Corollary 2.3 Let $\lambda / \mu$ be an edgewise connected skew shape with d diagonals. Then $\lambda / \mu$ has $2^{d-1}$ outside decompositions.

The determinantal formula of Hamel and Goulden relies on the operation \# on border strips of an outside decomposition. We will show that the \# operation can be described in terms of the cutting strip of the outside decomposition. To this end, we need to record the contents of the diagonals in the construction of the cutting strip. For example, the cutting strip in figure 3 inherits the contents of the boxes in the original shape.

Given an outside decomposition $\Pi$ of a skew shape $\lambda / \mu$ and a strip $\theta$ in $\Pi$, let $\phi$ be the cutting strip of $\Pi$. We denote the content of the starting box of $\theta$ and the content of the
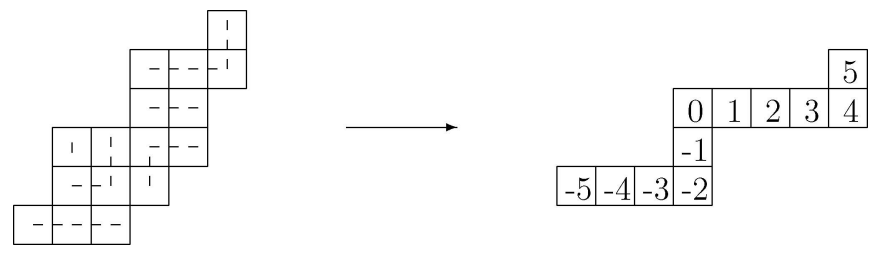

Figure 3. Contents of a cutting strip. 
ending box of $\theta$ respectively by $p(\theta)$ and $q(\theta)$, then $\theta$ forms a segment of the cutting strip of $\Pi$ starting with the box having content $p(\theta)$ and ending with the box with content $q(\theta)$, which is denoted by $\phi[p(\theta), q(\theta)]$, or simply $[p(\theta), q(\theta)]$ if no confusion arises. Note that the contents of the cutting strip are inherited from the contents in the original skew shape. Furthermore, we may extend the notation $\phi[p, q]$ by the following convention:

(1) if $p \leq q$, then $\phi[p, q]$ is a segment of $\phi$ starting with the box having content $p$ and ending with the box having content $q$;

(2) $\phi[q+1, q]=\varnothing$;

(3) if $p>q+1$, then $\phi[p, q]$ is undefined.

The difference between an empty strip and an undefined strip lies in values of the corresponding Schur functions. For an empty strip, the corresponding Schur function is defined to be 1, and for the undefined strip, the Schur function takes the value 0. Next we observe that the \# operation on strips can be described as extracting segments of the cutting strip. The proof of the following fact is omitted since it is merely a technical verification.

Theorem 2.4 Let $\lambda / \mu$ be an edgewise connected skew shape, and $\Pi$ be an outside decomposition of $\lambda / \mu$. For any strips $\theta_{i}, \theta_{j}$ in $\Pi$, the strip $\theta_{i} \# \theta_{j}$, as defined in [7], can be represented as $\left[p\left(\theta_{j}\right), q\left(\theta_{i}\right)\right]$.

For example, the strips in figure 3 are $\theta_{1}=33 / 2, \theta_{2}=2, \theta_{3}=21, \theta_{4}=22 / 1, \theta_{5}=1$, $\theta_{6}=3$, and some of the strips obtained by the \# operation are given below (see figure 4):

$$
\begin{array}{ll}
\theta_{1} \# \theta_{5}=[-2,5]=5511 / 4, & \theta_{5} \# \theta_{1}=[2,-2]=\text { undefined, } \\
\theta_{2} \# \theta_{3}=[-1,2]=31, & \theta_{3} \# \theta_{2}=[1,1]=1, \\
\theta_{4} \# \theta_{6}=[-5,-1]=44 / 3, & \theta_{6} \# \theta_{4}=[-3,-3]=1 .
\end{array}
$$

\section{Transformations of determinants}

This section is concerned with a transformation theorem on the determinantal formula of Hamel and Goulden [7]. In the proof of the rim ribbon formula for Schur functions, Lascoux and Pragacz give a transformation from the Giambelli determinant to the rim

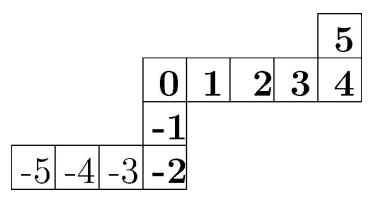

Figure 4. $\theta_{1} \# \theta_{5}$ as a segment $[-2,5]$ of the cutting strip. 
ribbon determinant. Since the Hamel-Goulden determinant is a unification of the four known determinantal identities, it is natural to consider the transformation on the HamelGoulden determinants, namely, from one outside decomposition to another. From the cutting strip characterization of outside decompositions, it follows that one can obtain any outside decomposition from another by a sequence of basic transformations of changing the directions of the boxes on a diagonal, which corresponds to the operation of changing the direction of a box in the cutting strip. This transformation is called the twist transformation on border strips. Specifically, we use $\omega_{i}$ to denote the twist transformation acting on an outside decomposition $\Pi$ by changing the directions of the diagonal boxes with content $i$.

We may restrict our attention to the determinantal transformations that correspond to the twist transformations on the cutting strips, or equivalently on the outside decompositions. Let us review the Hamel-Goulden determinantal formula. Without loss of generality, we may assume that $\lambda / \mu$ is an edgewise connected skew shape with $d$ diagonals. Let $\Pi=$ $\left(\theta_{1}, \theta_{2}, \ldots, \theta_{m}\right)$ be an outside decomposition of $\lambda / \mu$. Then we have

Theorem 3.1 (Hamel and Goulden [7]) The skew Schurfunction $s_{\lambda / \mu}(X)$ can be evaluated by the following determinant:

$$
D(\Pi)=\operatorname{det}\left[\begin{array}{cccc}
s_{\theta_{1}} \# \theta_{1} & s_{\theta_{1} \# \theta_{2}} & \ldots & s_{\theta_{1} \# \theta_{m}} \\
s_{\theta_{2}} \# \theta_{1} & s_{\theta_{2}} \# \theta_{2} & \ldots & s_{\theta_{2}} \# \theta_{m} \\
\vdots & \vdots & \ldots & \vdots \\
s_{\theta_{m} \# \theta_{1}} & s_{\theta_{m}} \# \theta_{2} & \ldots & s_{\theta_{m}} \# \theta_{m}
\end{array}\right],
$$

where $s_{\varnothing}=1$ and $s_{\theta_{i}} \# \theta_{j}=0$ if $\theta_{i} \# \theta_{j}$ is undefined.

We recall that the above determinantal formula includes the Jacobi-Trudi identity, the dual Jacobli-Trudi identity (the Nägelsbach-Kostka identity), the Giambelli identity and the rim ribbon identity as special cases. Note that for any order of the strips in the outside decomposition, the above determinant remains invariant. The corresponding cutting strips for the above special cases are the horizontal strip, the vertical strip, the maximal hook, and the maximal outside rim ribbon, see [7]. As noticed by Lascoux and Pragacz [9], one may obtain the rim ribbon identity from the Giambelli identity, and vice versa. We may expect that the above mentioned four identities can be transformed from one to another. In fact, this goal can be achieved in a more general setting in terms of the transformation theorem on the Hamel-Goulden identity.

For an outside decomposition $\Pi=\left(\theta_{1}, \theta_{2}, \ldots, \theta_{m}\right)$ of $\lambda / \mu$, we define its inversion number by

$$
\operatorname{inv}(\Pi)=\left|\left\{(i, j) ; p\left(\theta_{i}\right)>p\left(\theta_{j}\right), q\left(\theta_{i}\right)<q\left(\theta_{j}\right)\right\}\right| .
$$


By the cutting strip characterization of the \# operation, we may rewrite (3.1) as

$$
(-1)^{\operatorname{inv}(\Pi)} \operatorname{det}\left[\begin{array}{cccc}
s_{\left[p_{1}, q_{1}\right]} & s_{\left[p_{2}, q_{1}\right]} & \ldots & s_{\left[p_{m}, q_{1}\right]} \\
s_{\left[p_{1}, q_{2}\right]} & s_{\left[p_{2}, q_{2}\right]} & \ldots & s_{\left[p_{m}, q_{2}\right]} \\
\vdots & \vdots & \ldots & \vdots \\
s_{\left[p_{1}, q_{m}\right]} & s_{\left[p_{2}, q_{m}\right]} & \ldots & s_{\left[p_{m}, q_{m}\right]}
\end{array}\right],
$$

where $p_{1}>p_{2}>\cdots>p_{m}$ are the rearrangement of $p\left(\theta_{1}\right), \ldots, p\left(\theta_{m}\right)$ in decreasing order and $q_{i}=q\left(\theta_{i}\right)$ for $1 \leq i \leq m$. Since $p_{1}>p_{2}>\cdots>p_{m}$ and $q_{1}>q_{2}>\cdots>$ $q_{m}$, it is clear that if $s_{\left[p_{i}, q_{j}\right]}=0$ then $s_{\left[p_{i}, q_{j^{\prime}}\right]}=0$ and $s_{\left[p_{i^{\prime}}, q_{j}\right]}=0$ for $j \leq j^{\prime} \leq m$ and $1 \leq i^{\prime} \leq i$. The above canonical form is more convenient for the construction of the determinantal transformations corresponding to the twist transformations on outside decompositions.

Now the question becomes how to transform the determinant (3.2) for an outside decomposition $\Pi$ to the determinant corresponding to the outside decomposition $\omega_{i}(\Pi)$. To accomplish this goal, we need an important product rule for the skew Schur functions due to Zelevinsky [17], which follows from the Jeu de Taquin of Schützenberger [13], and can be verified by the combinatorial definition. This product rule is also used by Lascoux and Pragacz [9] in their transformation from the Giambelli identity to the rim ribbon identity.

Given two skew diagrams $I$ and $J, I \triangleright J$ is the diagram obtained by gluing the lower left-hand corner box of diagram $J$ to the right of the upper right-hand corner box of diagram $I$ and $I \uparrow J$ is the diagram obtained by gluing the lower left-hand corner box of diagram $J$ to the top of the upper right-hand corner box of diagram $I$. For example, if $I=32 / 1$ and $J=332 / 2$, then $I \triangleright J$ and $I \uparrow J$ are given by figure 5 .

Lemma 3.2 Let I and $J$ be two skew diagrams. Then

$$
s_{I} s_{J}=s_{I \triangleright J}+s_{I \uparrow J} .
$$

As far as this paper is concerned, we will only need the above property for ribbon Schur functions, or the strip Schur functions. More specifically, we need to multiply a row of the Hamel-Goulden determinant by a ribbon Schur function and subtract the product from another row to obtain a new ribbon Schur function.
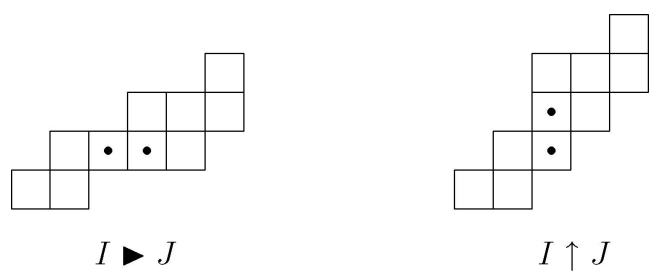

Figure 5. The $\boldsymbol{\square}$ and $\uparrow$ operator for skew diagrams. 
For two strips $\alpha$ and $\beta$, one has two ways to combine them into another ribbon, one is $\alpha \triangleright \beta$ and the other is $\alpha \uparrow \beta$. It turns out that the basic transformation on the cutting strip is an interchange of the operations $\alpha \triangleright \beta$ and $\alpha \uparrow \beta$. This is the reason why we call $\omega_{i}$ the twist transformation.

Theorem 3.3 If $\lambda / \mu$ is an edgewise connected skew shape with d diagonals, let $\Pi$ be an outside decomposition of $\lambda / \mu$, then for each diagonal of $\lambda / \mu$ with content $i$, we can obtain $D\left(\omega_{i}(\Pi)\right)$ from $D(\Pi)$ by elementary determinantal operations.

We remark that the elementary determinantal operations mentioned in the above theorem actually involve three types, i.e., interchanging two rows or columns; multiplying a row or a column by a non-zero scalar, or adding a multiple of one row or one column to another row or column; and from two determinants, one can construct a bigger determinant by forming a bigger diagonal block matrix, or one can reduce a diagonal determinant into two smaller determinants, one of which may be the identity matrix.

Note that if $L$ is a diagonal of an edgewise connected skew diagram $\lambda / \mu$, then there are four possible diagonal types classified by whether the first diagonal box has a box on the top, and whether the last diagonal box has a box on the right. These four types are depicted by figure 6 .

We now proceed to give the proof of Theorem 3.3. There are essentially two different types (Type 1 and Type 3), because Type 2 is similar to Type 1 , and Type 4 is similar to Type 3 . For Type 1 and Type 2 diagonals, the determinantal transformations involve changing a determinant to another determinant of different order. We need the following lemma for counting the number of inversions of a sequence of pairs of numbers, which will be used to deal with the sign change of determinants after permuting rows and columns. Given a sequence of $m$ pairs $P=\left\{\left(a_{1}, b_{1}\right),\left(a_{2}, b_{2}\right), \ldots,\left(a_{m}, b_{m}\right)\right\}$ with $a_{1}, a_{2}, \ldots, a_{m}$ being distinct and $b_{1}, b_{2}, \ldots, b_{m}$ being distinct. We define the inversion number of the sequence by

$$
\operatorname{inv}(P)=\left|\left\{(i, j) ; a_{i}>a_{j}, b_{i}<b_{j}\right\}\right|
$$

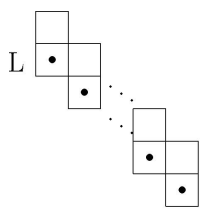

Type 1

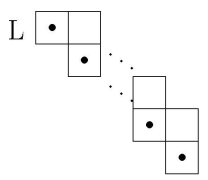

Type 3

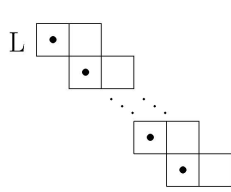

Type 2

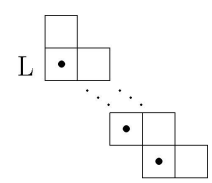

Type 4

Figure 6. Four possible types of diagonals of $\lambda / \mu$. 
It can be easily seen that the inversion number depends only on the set of pairs in a sequence. We choose the sequence notation for the sake of presentation.

Lemma 3.4 Let $P=\left\{\left(a_{1}, b_{1}\right),\left(a_{2}, b_{2}\right), \ldots,\left(a_{m}, b_{m}\right)\right\}$ be a sequence of $m$ pairs such that $a_{1}, a_{2}, \ldots, a_{m}$ are distinct and $b_{1}, b_{2}, \ldots, b_{m}$ are distinct. Let $\sigma$ be a permutation of $1,2, \ldots, m$ acting on the indices of $a_{1}, a_{2}, \ldots, a_{m}, \sigma\left(a_{i}\right)=a_{\sigma(i)}$, then we have

$$
\operatorname{inv}\left(\left\{\left(\sigma\left(a_{1}\right), b_{1}\right),\left(\sigma\left(a_{2}\right), b_{2}\right), \ldots,\left(\sigma\left(a_{m}\right), b_{m}\right)\right\}\right) \equiv \operatorname{inv}(\sigma)+\operatorname{inv}(P)(\bmod 2) .
$$

Proof: Suppose $b_{i_{1}}<b_{i_{2}}<\cdots<b_{i_{m}}$ is the reordering of $b_{1}, b_{2}, \ldots, b_{m}$, then we have

$$
\operatorname{inv}(P)=\operatorname{inv}\left(\left(a_{i_{1}}, a_{i_{2}}, \ldots, a_{i_{m}}\right)\right)=\left|\left\{(k, l) ; a_{i_{k}}>a_{i_{l}}, k<l\right\}\right| .
$$

and

$$
\begin{aligned}
& \operatorname{inv}\left(\left\{\left(\sigma\left(a_{1}\right), b_{1}\right),\left(\sigma\left(a_{2}\right), b_{2}\right), \ldots,\left(\sigma\left(a_{m}\right), b_{m}\right)\right\}\right) \\
& \quad=\operatorname{inv}\left(\left(a_{\sigma\left(i_{1}\right)}, a_{\sigma\left(i_{2}\right)}, \ldots, a_{\sigma\left(i_{m}\right)}\right)\right) \\
& \quad \equiv \operatorname{inv}(\sigma)+\operatorname{inv}\left(\left(a_{i_{1}}, a_{i_{2}}, \ldots, a_{i_{n}}\right)\right)(\bmod 2) \\
& \quad \equiv \operatorname{inv}(\sigma)+\operatorname{inv}(P)(\bmod 2) .
\end{aligned}
$$

This completes the proof.

We are now ready to present the proof of Theorem 3.3 for Type 1 diagonals. Let $\Pi=$ $\left(\theta_{1}, \theta_{2}, \ldots, \theta_{m}\right)$ be an outside decomposition of an edgewise connected skew diagram $\lambda / \mu$, and let $\phi$ be the cutting strip of $\Pi$. Recall that the sequence of the contents of the starting boxes of the strips in $\Pi$ is $p_{1}>p_{2}>\cdots>p_{m}$ and the sequence of the contents of the ending boxes of the strips in $\Pi$ is $q_{1}>q_{2}>\cdots>q_{m}$.

Suppose that $\lambda / \mu$ has a Type 1 diagonal $L$ with content $i$, and suppose that there are $r$ boxes in $L$. We may assume without loss of generality that the diagonal boxes all go up, since we may reverse the transformation process for the case that the diagonal boxes all go right. Thus the cutting strip of $\Pi$ may be written as $\left[p_{m}, i\right] \uparrow\left[i+1, q_{1}\right]$ and the cutting strip of $\omega_{i}(\Pi)$ may be written as $\left[p_{m}, i\right] \triangleright\left[i+1, q_{1}\right]$. Notice that the twist transformation $\omega_{i}$ does not change the contents of the cutting strip of $\Pi$.

Since $\omega_{i}$ only changes the strips which contain a box in $L$, we may suppose that $\theta_{i}$, $1 \leq t \leq r$, is the strip in $\Pi$ that contains the $t$-th diagonal box in $L$. Then we have $p\left(\theta_{i_{t}}\right) \leq i<q\left(\theta_{i_{t}}\right)$, for $1 \leq t \leq r$. As illustrated in figure 7 , under the operation $\omega_{i}$, the strip

$$
\theta_{i_{1}}=\left[p\left(\theta_{i_{1}}\right), q\left(\theta_{i_{1}}\right)\right]=\left[p\left(\theta_{i_{1}}\right), i\right] \uparrow\left[i+1, q\left(\theta_{i_{1}}\right)\right]
$$

breaks into two strips

$$
\left[p\left(\theta_{i_{1}}\right), q\left(\theta_{i_{2}}\right)\right]=\left[p\left(\theta_{i_{1}}\right), i\right] \bullet\left[i+1, q\left(\theta_{i_{2}}\right)\right] \quad \text { and } \quad\left[i+1, q\left(\theta_{i_{1}}\right)\right] .
$$




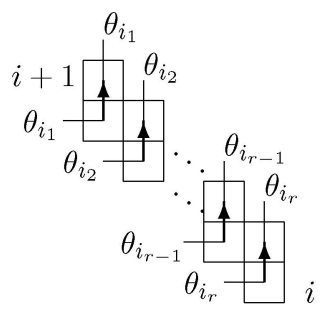

$L$

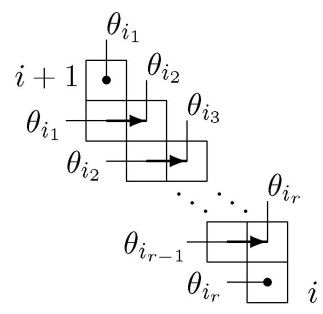

$\omega_{i}(L)$

Figure 7. $\omega_{i}$ acts on a Type 1 diagonal $L$.

If $r>1$ then the last strip

$$
\theta_{i_{r}}=\left[p\left(\theta_{i_{r}}\right), q\left(\theta_{i_{r}}\right)\right]=\left[p\left(\theta_{i_{r}}\right), i\right] \uparrow\left[i+1, q\left(\theta_{i_{r}}\right)\right]
$$

will be cut off into $\left[p\left(\theta_{i_{r}}\right), i\right]$, and the other strips

$$
\theta_{i_{t}}=\left[p\left(\theta_{i_{t}}\right), q\left(\theta_{i_{t}}\right)\right]=\left[p\left(\theta_{i_{t}}\right), i\right] \uparrow\left[i+1, q\left(\theta_{i_{t}}\right)\right], 2 \leq t \leq r-1,
$$

will be twisted into

$$
\left[p\left(\theta_{i_{t}}\right), q\left(\theta_{i_{t+1}}\right)\right]=\left[p\left(\theta_{i_{t}}\right), i\right] \triangleright\left[i+1, q\left(\theta_{i_{t+1}}\right)\right] .
$$

By Lemma 3.4 one can verify that $\operatorname{inv}\left(\omega_{i}(\Pi)\right) \equiv \operatorname{inv}(\Pi)(\bmod 2)$.

Since $p\left(\theta_{i_{t}}\right)<i+1$ for $1 \leq t \leq r$, there is a unique integer $k, 1 \leq k \leq m-r+1$, such that $p_{k}<i+1$ and $p_{k-1}>i+1$ where $p_{0}$ is defined to be $\infty$, if necessary. Therefore, there are exactly $k-1$ strips in $\Pi$ for which the contents of starting boxes are bigger than $i+1$, and there are exactly $k-1+r$ strips in $\Pi$ for which the contents of ending boxes are bigger than $i$. It follows that $q_{k+r-1}>i$ and $q_{k+r}<i$ if $k+r<m+1$. Moreover, the sequence of the contents of the starting boxes of the strips in $\omega_{i}(\Pi)$ becomes $p_{1}>\cdots>p_{k-1}>i+1>p_{k}>\cdots>p_{m}$, and the sequence of the contents of the ending boxes of the strips in $\omega_{i}(\Pi)$ becomes $q_{1}>\cdots>q_{k+r-1}>i>q_{k+r}>\cdots>q_{m}$. As noted before, the determinant $D(\Pi)$ has the following canonical form:

$$
(-1)^{\operatorname{inv}(\Pi)} \operatorname{det}\left[\begin{array}{ll}
A & B \\
0 & C
\end{array}\right]
$$


where

$$
\begin{aligned}
& A=\left[\begin{array}{ccc}
s_{\left[p_{1}, q_{1}\right]} & \cdots & s_{\left[p_{k-1}, q_{1}\right]} \\
\vdots & \cdots & \vdots \\
s_{\left[p_{1}, q_{k+r-1}\right]} & \cdots & s_{\left[p_{k-1}, q_{k+r-1}\right]}
\end{array}\right]_{(k+r-1) \times(k-1)}, \\
& B=\left[\begin{array}{ccc}
s_{\left[p_{k}, i\right] \uparrow\left[i+1, q_{1}\right]} & \cdots & s_{\left[p_{m}, i\right] \uparrow\left[i+1, q_{1}\right]} \\
\vdots & \ldots & \vdots \\
s_{\left[p_{k}, i\right] \uparrow\left[i+1, q_{k+r-1}\right]} & \cdots & s_{\left[p_{m}, i\right] \uparrow\left[i+1, q_{k+r-1}\right]}
\end{array}\right]_{(k+r-1) \times(m-k+1)}, \\
& C=\left[\begin{array}{ccc}
s_{\left[p_{k}, q_{k+r}\right]} & \cdots & s_{\left[p_{m}, q_{k+r}\right]} \\
\vdots & \cdots & \vdots \\
s_{\left[p_{k}, q_{m}\right]} & \cdots & s_{\left[p_{m}, q_{m}\right]}
\end{array}\right]_{(m-k-r+1) \times(m-k+1)} .
\end{aligned}
$$

Using the sequences of the contents of starting boxes and ending boxes of the strips of $\omega_{i}(\Pi)$, we may obtain the following canonical form of $D\left(\omega_{i}(\Pi)\right)$ :

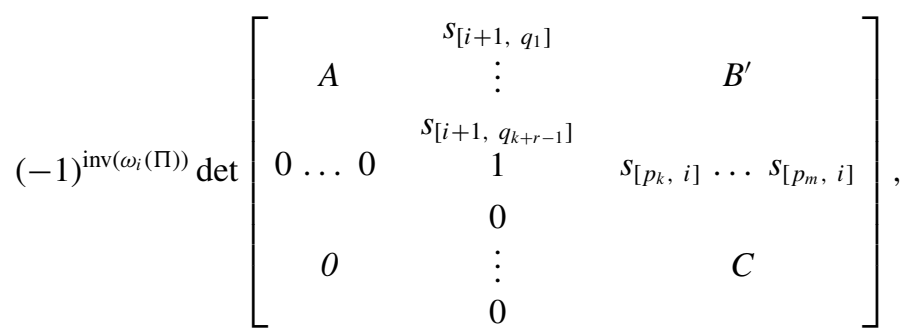

where

$$
B^{\prime}=\left[\begin{array}{ccc}
s_{\left[p_{k}, i\right] \triangleright\left[i+1, q_{1}\right]} & \cdots & s_{\left[p_{m}, i\right] \triangleright\left[i+1, q_{1}\right]} \\
\vdots & \cdots & \vdots \\
s_{\left[p_{k}, i\right] \triangleright\left[i+1, q_{k+r-1}\right]} & \cdots & s_{\left[p_{m}, i\right] \triangleright\left[i+1, q_{k+r-1}\right]}
\end{array}\right]_{(k+r-1) \times(m-k+1)}
$$

Now we need to transform (3.3) into the following determinant by adding a row and a column as shown below. This operation involves a sign $(-1)^{2 k+r}$ :

$$
(-1)^{\operatorname{inv}(\Pi)} \operatorname{det}\left[\begin{array}{cccc} 
& 0 & \\
A & \vdots & B \\
& 0 & \\
0 \ldots & 1 & s_{\left[p_{k}, i\right]} \ldots & s_{\left[p_{m}, i\right]} \\
& 0 & & \\
0 & \vdots & C
\end{array}\right]
$$


Applying Lemma 3.2, we have

$$
s_{\left[p_{t}, i\right] \triangleright\left[i+1, q_{l}\right]}=s_{\left[p_{t}, i\right]} S_{\left[i+1, q_{l}\right]}-s_{\left[p_{t}, i\right] \uparrow\left[i+1, q_{l}\right]}
$$

for $k \leq t \leq m$ and $1 \leq l \leq k+r-1$. The above identity enables us to use the $(k+r)$-th row of (3.5) to twist the entries in $B$ by elementary determinantal operations. Such operations will change $B$ into $-B^{\prime}$. Multiplying the last $m-k+2$ columns and the last $m-k-r+2$ rows of (3.5) by -1 , we may derive (3.4) from (3.5). It follows that we can transform $D(\Pi)$ into $D\left(\omega_{i}(\Pi)\right)$ by elementary determinantal operations. This completes the proof of Theorem 3.3 for Type 1 diagonals.

We next consider the case when $\lambda / \mu$ has a Type 3 diagonal $L$ with content $i$. Suppose that there are $r$ boxes in $L$ and suppose that the directions of the diagonal boxes of $L$ all go up. Thus, the cutting strip of $\Pi$ may be written as $\left[p_{m}, i\right] \uparrow\left[i+1, q_{1}\right]$ and the cutting strip of $\omega_{i}(\Pi)$ may be written as $\left[p_{m}, i\right] \triangleright\left[i+1, q_{1}\right]$. We assume that $\theta_{i_{t}}, 1 \leq t \leq r$, is the strip in $\Pi$ that contains the $t$-th diagonal box in $L$. Then we have $p\left(\theta_{i_{t}}\right) \leq i \leq q\left(\theta_{i_{t}}\right)$, for $1 \leq t \leq r$. As illustrated in figure 8, under the operation $\omega_{i}$, the first strip $\theta_{i_{1}}=\left[p\left(\theta_{i_{1}}\right), i\right]$ equals

$$
\left[p\left(\theta_{i_{1}}\right), q\left(\theta_{i_{2}}\right)\right]=\left[p\left(\theta_{i_{1}}\right), i\right] \triangleright\left[i+1, q\left(\theta_{i_{2}}\right)\right] .
$$

If $r>1$, the last strip

$$
\theta_{i_{r}}=\left[p\left(\theta_{i_{r}}\right), q\left(\theta_{i_{r}}\right)\right]=\left[p\left(\theta_{i_{r}}\right), i\right] \uparrow\left[i+1, q\left(\theta_{i_{r}}\right)\right]
$$

will be cut off into $\left[p\left(\theta_{i_{r}}\right), i\right]$, and the other strips

$$
\theta_{i_{t}}=\left[p\left(\theta_{i_{t}}\right), q\left(\theta_{i_{t}}\right)\right]=\left[p\left(\theta_{i_{t}}\right), i\right] \uparrow\left[i+1, q\left(\theta_{i_{t}}\right)\right],
$$

will be twisted into

$$
\left[p\left(\theta_{i_{t}}\right), q\left(\theta_{i_{t+1}}\right)\right]=\left[p\left(\theta_{i_{t}}\right), i\right] \triangleright\left[i+1, q\left(\theta_{i_{t+1}}\right)\right], 2 \leq t \leq r-1 .
$$

By Lemma 3.4 one may verify that $\operatorname{inv}\left(\omega_{i}(\Pi)\right) \equiv \operatorname{inv}(\Pi)+r-1(\bmod 2)$.

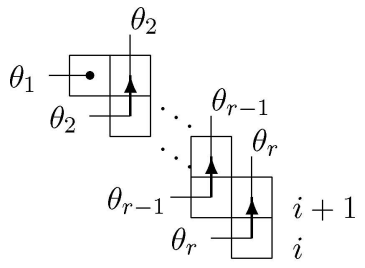

$L$

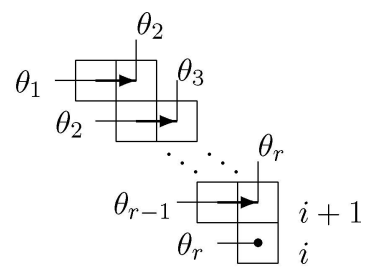

$\omega_{i}(L)$

Figure 8. $\omega_{i}$ acts on a Type 3 diagonal $L$. 
Since $i$ is the content of the ending box of $\theta_{i_{1}}$ and $q\left(\theta_{i_{t}}\right) \geq i$ for $1 \leq t \leq r$, there is a unique integer $k, 1 \leq k \leq m-r+1$ such that $q_{k}=i$. So we have $p_{k-r+1}<i+1$ and $p_{k+r}>i+1$ if $k+r<m+1$. Note that in this case $D(\Pi)$ still has the canonical form

$$
(-1)^{\operatorname{inv}(\Pi)} \operatorname{det}\left[\begin{array}{ll}
E & F \\
0 & G
\end{array}\right],
$$

where

$$
\begin{aligned}
& E=\left[\begin{array}{ccc}
s_{\left[p_{1}, q_{1}\right]} & \cdots & s_{\left[p_{k-r}, q_{1}\right]} \\
\vdots & \cdots & \vdots \\
s_{\left[p_{1}, q_{k-1}\right]} & \cdots & s_{\left[p_{k-r}, q_{k-1}\right]} \\
0 & \cdots & 0
\end{array}\right]_{k \times(k-r)}, \\
& F=\left[\begin{array}{ccc}
s_{\left[p_{k-r+1}, i\right] \uparrow\left[i+1, q_{1}\right]} & \cdots & s_{\left.\left[p_{m}, i\right] \uparrow\left[i+1, q_{1}\right)\right]} \\
\vdots & \ldots & \vdots \\
s_{\left[p_{k-r+1}, i\right] \uparrow\left[i+1, q_{k-1}\right]} & \cdots & s_{\left[p_{m}, i\right] \uparrow\left[i+1, q_{k-1}\right]} \\
s_{\left[p_{k-r+1}, i\right]} & \cdots & s_{\left[p_{m}, i\right]}
\end{array}\right]_{k \times(m-k+r)} \text {, } \\
& G=\left[\begin{array}{ccc}
s_{\left[p_{k-r+1}, q_{k+1}\right]} & \cdots & s_{\left[p_{m}, q_{k+1}\right]} \\
\vdots & \ldots & \vdots \\
s_{\left[p_{k-r+1}, q_{m}\right]} & \cdots & s_{\left[p_{m}, q_{m}\right]}
\end{array}\right]_{(m-k) \times(m-k+r)} .
\end{aligned}
$$

On the other hand, $D\left(\omega_{i}(\Pi)\right)$ has the following canonical form:

$$
(-1)^{\operatorname{inv}\left(\omega_{i}(\Pi)\right)} \operatorname{det}\left[\begin{array}{ll}
E & F^{\prime} \\
0 & G
\end{array}\right]
$$

where

$$
F^{\prime}=\left[\begin{array}{ccc}
s_{\left[p_{k-r+1}, i\right] \triangleright\left[i+1, q_{1}\right]} & \ldots & s_{\left.\left[p_{m}, i\right] \triangleright\left[i+1, q_{1}\right)\right]} \\
\vdots & \ldots & \vdots \\
s_{\left[p_{k-r+1}, i\right] \triangleright\left[i+1, q_{k-1}\right]} & \ldots & s_{\left[p_{m}, i\right] \triangleright\left[i+1, q_{k-1}\right]} \\
s_{\left[p_{k-r+1}, i\right]} & \cdots & s_{\left[p_{m}, i\right]}
\end{array}\right]_{k \times(m-k+r)}
$$

Applying Lemma 3.2 we get

$$
s_{\left[p_{t}, i\right] \triangleright\left[i+1, q_{l}\right]}=s_{\left[p_{t}, i\right]} S_{\left[i+1, q_{l}\right]}-s_{\left[p_{t}, i\right] \uparrow\left[i+1, q_{l}\right]}
$$

for $k-r+1 \leq t \leq m$ and $1 \leq l \leq k-1$. So, we may change $F$ into $-F^{\prime}$. Furthermore, by changing the signs of rows and columns, we obtain (3.7) from (3.6) with a sign $(-1)^{r+1}$. It 
follows that we can transform $D(\Pi)$ into $D\left(\omega_{i}(\Pi)\right)$ by elementary determinantal operations. Note that in this case there is no change on the order of the determinants.

The other two types of diagonals can be dealt with in a similar way. Hence we have completed the proof of Theorem 3.3.

For example, let $\lambda=5331$, and let $\Pi_{1}, \Pi_{2}, \Pi_{3}, \Pi_{4}$ be the outside decompositions of $\lambda$ with respect to the Jacobi-Trudi determinant, the Giambelli determinant, the LascouxPragacz determinant and the dual Jacobi-Trudi determinant. Then we have the following relations:

$$
\begin{aligned}
\omega_{-1} \omega_{-2} \omega_{-3}\left(\Pi_{1}\right) & =\Pi_{2}, \\
\omega_{1} \omega_{0} \omega_{-1} \omega_{-2}\left(\Pi_{2}\right) & =\Pi_{3}, \\
\omega_{3} \omega_{2} \omega_{-1} \omega_{-2}\left(\Pi_{3}\right) & =\Pi_{4} .
\end{aligned}
$$

So we may transform the Jacobi-Trudi determinant for $s_{5331}$ into the Giambelli determinant for $s_{5331}$, and transform the Giambelli determinant into the Lascoux-Pragacz determinant, and transform the Lascoux-Pragacz determinant to the dual Jacobi-Trudi determinant.

$$
\begin{aligned}
& \left|\begin{array}{cccc}
h_{5} & h_{6} & h_{7} & h_{8} \\
h_{2} & h_{3} & h_{4} & h_{5} \\
h_{1} & h_{2} & h_{3} & h_{4} \\
0 & 0 & 1 & h_{1}
\end{array}\right| \stackrel{\omega_{-3}}{\rightarrow}\left|\begin{array}{ccc}
s_{5} & s_{6} & s_{71} \\
s_{2} & s_{3} & s_{41} \\
s_{1} & s_{2} & s_{31}
\end{array}\right| \stackrel{\omega_{-2}}{\rightarrow}\left|\begin{array}{ccc}
s_{5} & s_{611} & s_{6} \\
s_{2} & s_{311} & s_{3} \\
s_{1} & s_{211} & s_{2}
\end{array}\right| \stackrel{\omega_{-1}}{\rightarrow}\left|\begin{array}{ccc}
s_{5111} & s_{51} & s_{5} \\
s_{2111} & s_{21} & s_{2} \\
s_{111} & s_{11} & s_{1}
\end{array}\right| \\
& \stackrel{\omega_{-2}}{\rightarrow}\left|\begin{array}{ccc}
s_{51} & s_{621 / 1} & s_{5} \\
s_{21} & s_{321 / 1} & s_{2} \\
s_{11} & s_{221 / 1} & s_{1}
\end{array}\right| \stackrel{\omega_{-1}}{\rightarrow}\left|\begin{array}{ccc}
s_{5} & s_{6} & s_{71} \\
s_{2} & s_{3} & s_{41} \\
s_{1} & s_{2} & s_{31}
\end{array}\right| \stackrel{\omega_{0}}{\rightarrow}\left|\begin{array}{ccc}
s_{52 / 1} & s_{631 / 2} & s_{41} \\
s_{22 / 1} & s_{331 / 2} & s_{11} \\
s_{2} & s_{31} & s_{1}
\end{array}\right| \\
& \stackrel{\omega_{1}}{\rightarrow}\left|\begin{array}{ccc}
s_{5331 / 22} & s_{422 / 11} & s_{311} \\
s_{331 / 2} & s_{22 / 1} & s_{11} \\
s_{31} & s_{2} & s_{1}
\end{array}\right| \stackrel{\omega_{-2}}{\rightarrow}\left|\begin{array}{ccc}
s_{422 / 11} & s_{42211 / 11} & s_{311} \\
s_{22 / 1} & s_{2211 / 1} & s_{11} \\
s_{2} & s_{211} & s_{1}
\end{array}\right| \\
& \stackrel{\omega_{-1}}{\rightarrow}\left|\begin{array}{ccc}
s_{311} & s_{3111} & s_{311111} \\
s_{11} & s_{111} & s_{1111} \\
s_{1} & s_{11} & s_{1111}
\end{array}\right| \stackrel{\omega_{2}}{\rightarrow}\left|\begin{array}{cccc}
s_{2} & s_{2111} & s_{21111} & s_{211111} \\
1 & s_{111} & s_{1111} & s_{111111} \\
0 & s_{11} & s_{111} & s_{1111} \\
0 & s_{1} & s_{11} & s_{1111}
\end{array}\right| \\
& \stackrel{\omega_{3}}{\rightarrow}\left|\begin{array}{ccccc}
e_{1} & e_{2} & e_{5} & e_{6} & e_{8} \\
1 & e_{1} & e_{4} & e_{5} & e_{7} \\
0 & 1 & e_{3} & e_{4} & e_{6} \\
0 & 0 & e_{2} & e_{3} & e_{5} \\
0 & 0 & e_{1} & e_{2} & e_{4}
\end{array}\right|
\end{aligned}
$$




\section{Supersymmetric Schur functions}

We note that Lemma 3.2 also holds for the supersymmetric Schur function $s_{\lambda / \mu}=s_{\lambda / \mu}(X, Y)$ with variable set in the form $X=\left\{\ldots, x_{-1}, x_{0}, x_{1}, \ldots\right\}$ and $Y=\left\{\ldots, y_{-1}, y_{0}, y_{1}, \ldots\right\}$. This fact can be verified by the tableaux representation of $s_{\lambda / \mu}(X, Y)$ given in [4] and the symmetric property of $s_{\lambda / \mu}(X, Y)$ in $X$ and $Y$. From the Jacobi-Trudi identity for the super Schur function $s_{\lambda / \mu}(X, Y)$ given in Goulden-Greene [4], and Macdonald [11], one can derive a determinantal formula analogous to Hamel and Goulden's identity for supersymmetric Schur functions. The lattice path interpretations can also be carried over to the supersymmetric case by using the weights given by Chen-Yan-Yang [1] in their study of the super Giambelli identity and the super Lascoux-Pragacz identity in the framework of the lattice path methodology. Formally speaking, we have the following theorem.

Theorem 4.1 For any outside decomposition $\Pi$ of a skew shape $\lambda / \mu$, let $D(\Pi)$ be the determinant in the form of (3.1) with the ribbon Schur function entries replaced by the supersymmetric ribbon Schur functions in the variable sets $X=\left\{\ldots, x_{-1}, x_{0}, x_{1}, \ldots\right\}$ and $Y=\left\{\ldots, y_{-1}, y_{0}, y_{1}, \ldots\right\}$. Then $D(\Pi)$ equals the supersymmetric Schur function $s_{\lambda / \mu}(X, Y)$.

\section{Acknowledgments}

We would like to thank Professor Alain Lascoux for valuable comments. This work was done under the auspices of the 973 Project on Mathematical Mechanization, the Ministry of Science and Technology, and the National Science Foundation of China.

\section{References}

1. W.Y.C. Chen, G.-G. Yan and A.L.B. Yang, “The skew Schubert polynomials," Europ. J. Combin. 25 (2004), 1181-1196.

2. Ö.N. Eğecioğlu and J.B. Remmel, “A combinatorial proof of the Giambelli identity for Schur functions,” $A d v$. Math. 70 (1988), 59-86.

3. M. Fulmek and C. Krattenthaler, "Lattice path proofs for determinantal formulas for symplectic and orthogonal characters," J. Combin. Theory. Ser. A 77 (1997), 3-50.

4. I.P. Goulden and C. Greene, "A new tableau representation for supersymmetric Schur functions," J. Alg. 170 (1994), 687-703.

5. I.M. Gessel and G. Viennot, "Binomial determinants, paths, and hook length formulae," Adv. Math. 58 (1985), 300-321.

6. I.M. Gessel and G. Viennot, "Determinants, paths, and plane partitions," preprint.

7. A.M. Hamel and I.P. Goulden, "Planar decompositions of tableaux and Schur function determinants," Europ. J. Combin. 16 (1995), 461-477.

8. A. Lascoux and P. Pragacz, "Équerres et fonctuons de Schur," C. R. Acad. Sci. Paris, Ser. I. Math. 299 (1984), 955-958.

9. A. Lascoux and P. Pragacz, "Ribbon Schur functions," Europ. J. Combin. 9 (1988), 561-574.

10. I.G. Macdonald, Symmetric Functions and Hall Polynomials, 2nd edition, Oxford University Press, Oxford, 1995.

11. I.G. Macdonald, Schur Functions: Theme and variations, Actes 28e Séminaire Lotharingien, Publ. I.R.M.A. Strasbourg, 1992, pp. 5-39. 
12. B.E. Sagan, The Symmetric Group, 2nd edition, Springer-Verlag Press, 2001.

13. M.P. Schützenberger, La correspondance de Robinson, in Combinatorics et représentation du groupe symmétrique (Table Ronde, Strasbourg 1976, D. Foata, ed.), Lecture Notes in Math., vol. 579, Springer, 1977, pp. 59-113.

14. R.P. Stanley, Enumerative Combinatorics, Vol. II, Cambridge University Press, 1999.

15. J.R. Stembridge, "Nonintersecting paths, Pfaffians, and plane partitions," Adv. Math. 83 (1990), 96-131.

16. K. Ueno, "Lattice path proof of the ribbon determinant formula for Schur functions," Nagoya Math. J. 124 (1991), 55-59.

17. A. Zelevinsky, "A generalization of the Littlewood-Richardson rule," J. Algebra 69 (1981), 82-94. 